\title{
B112 Simultaneous Velocity Measurement of Liquid-Solid Two-phase Flow by Using a PIV-PTV Combined System Yin CHEN $^{\circ}$, Tetsuo SAGA, Hui HU, Toshio KOBAYASHI , Shigeki SEGAWA, Nobuyuki TANIGUCHI PIV-PTVによる固液混相流の計測
}

陳 音、佐賀 徹雄、胡 暉、小林 敏雄、瀬川 茂樹、谷口 伸行 (東大)

\begin{abstract}
The velocities of both the continuos phase and the dispersed phase of the liquid-solid two-phase flow are measured simultaneously by PIV and PTV techniques, respectively. The fluorescent particles are used as the tracer particles for the fluid phase. A simultaneous image recording system is designed and the images of the tracer particles and the test particles are separated by the optical filters. The ensemble-averaged velocity and turbulent velocity fluctuation of both phases are analyzed and compared with the single-phase flow. The modification of the turbulence intensity of the fluid phase and the preferential concentration of the particles by turbulence are observed by the experiments.
\end{abstract}

Keywords: PIV, PTV, Simultaneous measurement, turbulent flow, Two-Phase Flow.

\section{INTRODUCTION}

Particle-laden flows are widely encountered in nature and in engineering applications. Particle dispersion and the turbulence modification in turbulent shear flows are important varieties of industrial energy processes and mixing processes, such as combustor, stirred tank reactor and cyclone separators. Recent development of numerical techniques enables us to carry out the detail information on the dispersion of the particles in the turbulent flow and the turbulence modulation by particles. However, there still maintain many discussions on the appropriate approach for the 2-way coupling model between fluid and dispersed phase. So it is necessary to measure the dispersed phase and the continuous phase simultaneously.

PIV represents an instantaneous whole field technique that makes it possible to detect spatial flow structure and provide a direct indication of the interphase coupling. In surveying recent literatures, several researches can be found that PIV were applied to the simultaneous measurement of both phases. For the multi-phase flow, the separation of the images of two phases is very important to the simultaneous measurement of the velocities of the two phases. The image separation methods can be classed into two groups, optical hardware based method and the digital image processing based method. The dyes and optical filters are commonly applied in the optical hardware based method to separate the particle images. For example, Suzuki, et al.[1] utilized two fluorescent dyes and suitable filter sets to separate tracer images from the others for the visualization of rising spheres in a quiescent fluid. Most of the former researches took advantage of the digital image processing based method. The common idea for this kind of method is separating the images of the two phases by the size of the images. Hishida, et al.[2], Suzuki, et al.[3] and Kiger[4] tried the simultaneous measurement for the particle-laden two-phase flow separated the two phase by this method. The main problem of the digital image processing method is that the spatial resolution of the analysis of the fluid phase is poor. Only the dilute concentrated flows can be measured and no carrier phase vectors could be detected near the location of the larger dispersed particles. The optical hardware based method just can avoid this problem.

In the present research, the fluorescent particles are utilized as the tracer of the fluid flow and a PIV-LIF combined image recording system is designed to capture the image of the tracer and test particles, separately. Considering the particle concentrations of 
the two kinds of the particles, we applied PIV and PTV techniques to the image processing of the continuos phase and dispersed phase, respectively.

\section{MEASUREMENT SYSTEM AND EXPERIMENT SETUP}

2.1 Image separating system

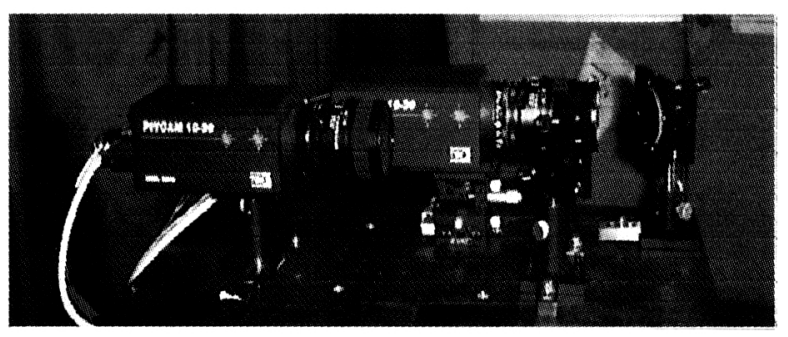

Figure 1. Recording System

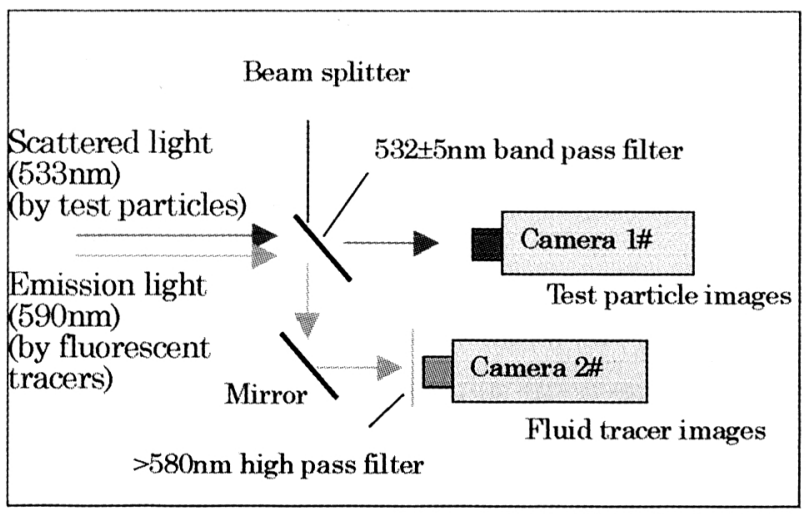

Figure 2. Principle of the image separating system
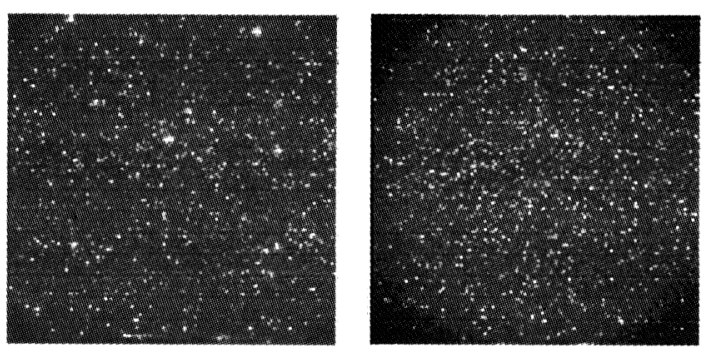

a. Test particle images b. Tracer particle images

Figure 3. Example of the original images

Figure 1 shows the photo of the image recording system. Figure 2 shows the principle of the image separating system. In the present study, Rhodamine B was used as fluorescent dye. It is known that the emission peak of Rhodamine $B$ is about $590 \mathrm{~nm}$, and the light scattered by the test particles is $532 \mathrm{~nm}$. As shown is Figure 1, a band pass filter ( $532 \pm 5 \mathrm{~nm}$ ) was installed in front of the Camera $1 \#$ and a high pass filter $(>580 \mathrm{~nm}$ pass) was installed at the head of the Camera
$2 \#$. By this recording system, the camera $1 \#$ record the image of the test particles which is not fluorescent and the camera $2 \#$ record the image of the fluorescent tracers, respectively. Figure 3 shows an example of the original images captured simultaneously by camera $1 \#$ and $2 \#$. It can be seen that the images of the two phases are separated successfully.

2.2 Experiment setup and measurement system

Figure 4 shows the schematically experiment setup used in the present research. The test circular nozzle $(\mathrm{D}=30 \mathrm{~mm})$ was fixed in the middle of the water tank $(600 \times 600 \times 1000 \mathrm{~mm})$. Fluorescent tracers $(20 \sim 40 \mu \mathrm{m})$ and test particles $(300 \mu \mathrm{m}$ and $500 \mu \mathrm{m}$, respectively) are premixed with water in a jet supply tank. The jet flow was supplied by a pump. The flowmeter was used to calculate the representative velocities and Reynolds numbers. A cylindrical plenum chamber with comb structures was installed at the upstream of the test nozzles to insure the turbulent levels of the core jet flows at the exit of test nozzle were less than $3 \%$.

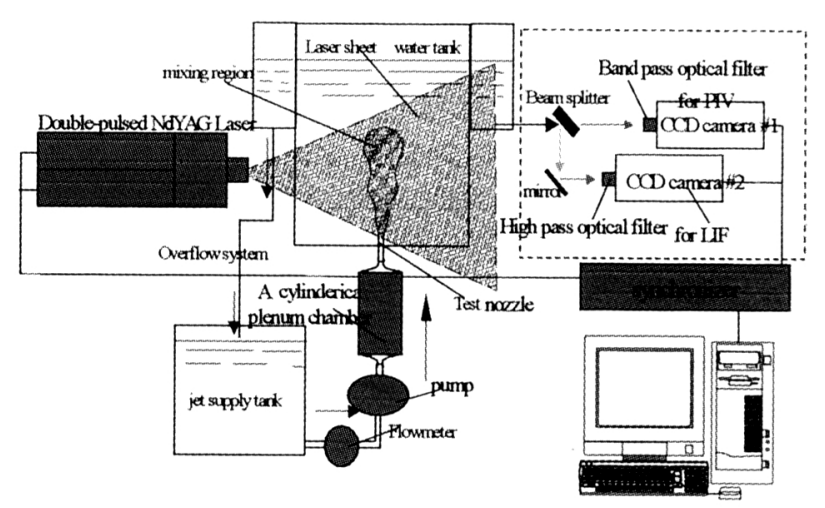

Figure 4. Experimental system setup

The measurement system consists of a doublepulsed Nd:YAG Laser system and 2 high-resolution CCD cameras. The wavelength of the light beams is $532 \mathrm{~nm}$. The laser beam was bundled in a planar laser sheet with thickness about $1.5 \mathrm{~mm}$. The frequency of the double-pulsed illumination is $15 \mathrm{~Hz}$ and power is $200 \mathrm{~mJ} /$ pulse. The Nd:YAG Laser and the simultaneous image recording cameras were connected to a host computer via a synchronizer which controls the timing of laser illumination and image acquisition. With 1GB RAM computer, 240 pairs of frames can be recorded every time.

The diameter of the circle nozzle was $D=30 \mathrm{~mm}$. The core jet velocity $\left(U_{0}\right)$ at the exit of the test nozzle was about $0.26 \mathrm{~m} / \mathrm{s}$. the Reynolds numbers based on the 


\section{Simultaneous Velocity Measurement of Liquid-Solid Two-phase Flow by Using a PIV- PTV Combined System}

nozzle exit diameter and the core jet velocities was about 8900 in the present study. The volume loading rate is about $0.03 \%$. The specific gravity of the test particles is 1.02 . Test area is $140 \times 140 \mathrm{~mm}$ at the exit of the nozzle.

\section{IMAGE PROCESSING}

The most widely used methods for PIV image processing can be classed into two categories, the particle tracking methods (PTV) and spatial correlation analysis methods (PIV), by the difference of the image densities. In the present research, because of fluorescent tracers are used to separate their images from the test particles, the tracers were put in with a relatively high concentration, that make it possible to apply the PIV method to the image processing of the fluid phase. And for relatively low concentrated dispersed phase, PTV method is applied.

\subsection{PIV image processing}

In the present research, an improved spatial correlation analysis method, named as Hierarchical Recursive PIV method[5], was used in the PIV image processing. The recent research shows that if a prior information of the local displacement is known, even by a smaller interrogation window could get the statistically meaningful PIV results. The first step of the method is as same as the conventional correlation analysis, but with relatively large interrogation window size and search range. The results of the first step were used as the approximate offset in the next step. By using the result of the former iteration step, the interrogation window size and the search range are reduced hierarchically. By this method, the spatial resolution can be increased with low error vector rate.

\subsection{PTV Image Processing}

In the present study, a correlation based Particle Tracking Velocimetry (PTV) algorithm (Saga, et al., [6]) was applied. The correlation between particle images and a Guassian particle mask was operated to improve the accuracy of particle locating up to sub-pixel level.

The path tracking of the test particles was achieved based on the correlation coefficient distribution of a small interrogation window around the studied particles at two time steps. The cross correlation between the gray pattern around the particle in the record of the first moment and the gray patterns around the particles within the search distance in the second moment was calculated. The particle of the second moment with maximum correlation coefficient value was determined as the same particle that in the first moment. Instead of the binary correlation, which is commonly used in the conventional PTV techniques, the gray pattern correlation was applied and the effect of the background noise was reduced.

\section{EXPERIMENTAL RESULTS AND DISCUSSION}
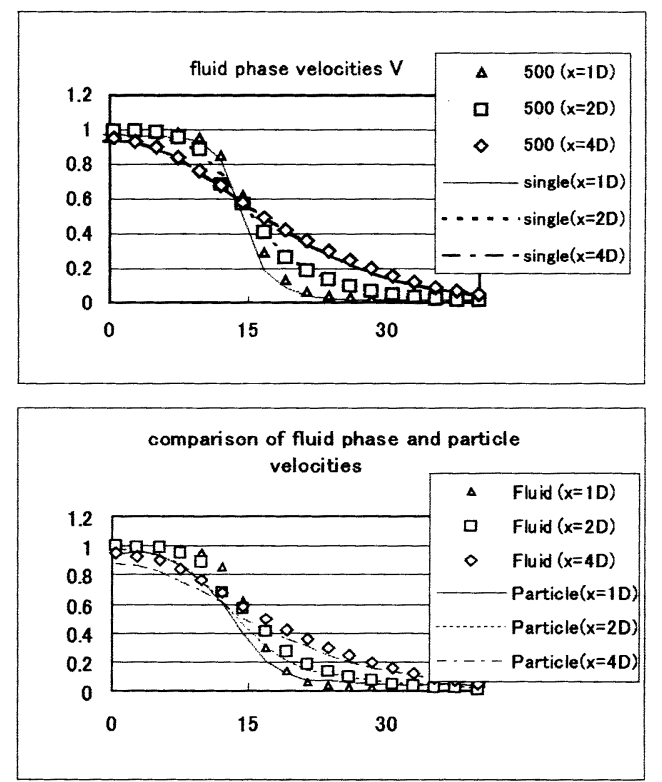

Figure 5. Ensemble-averaged velocities

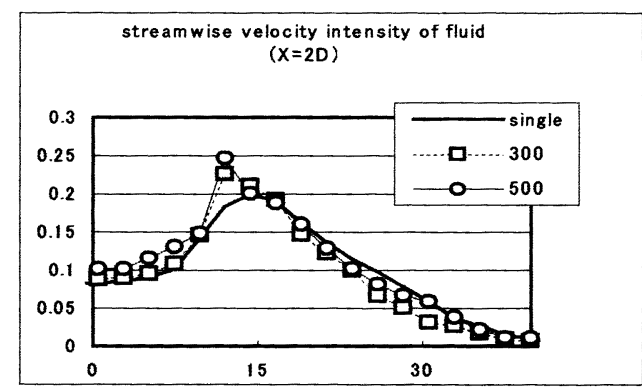

Figure 6. Streamwise turbulence intensities

Figure 5 and figure 6 show the ensemble-averaged velocity and turbulence intensity of both the continuous phase and the dispersed phase. The result of the single-phase flow is also shown to make the comparison. There is no obvious modification of the averaged velocity of the continuous phase when the larger test particles are added in the flow. However, loading of the test particles increases the turbulence intensities. It is agreed wiht the experiment result of channel flow by Hishida, et al. (1996). In the present experiment, the volume loading rate of the dispersed 
phase in only about $0.03 \%$, so we can consider that there is no effect from the collision of the particles, only the size and density of the particle are considered. The main reason of the intensity modification is that the particle size is larger than the Kolmogorov length scale, which is about $100 \mu \mathrm{m}$ at the exit of the nozzle.

Figure 7 shows the number density distribution of the test particles. The preferential concentration of the particles can be observed. The instantaneous result shown in figure 8 also illustrates the preferential concentration of the larger particles. The particles avoid the area with high shear rate, which is suggested by the previous simulations by Squires et al. [7].

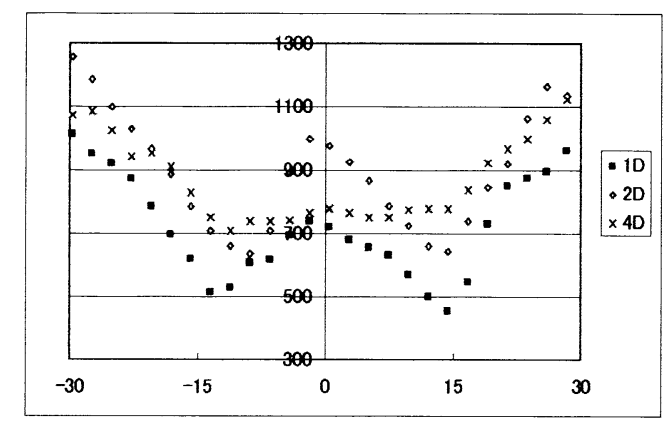

Figure 7. Particle number density distribution

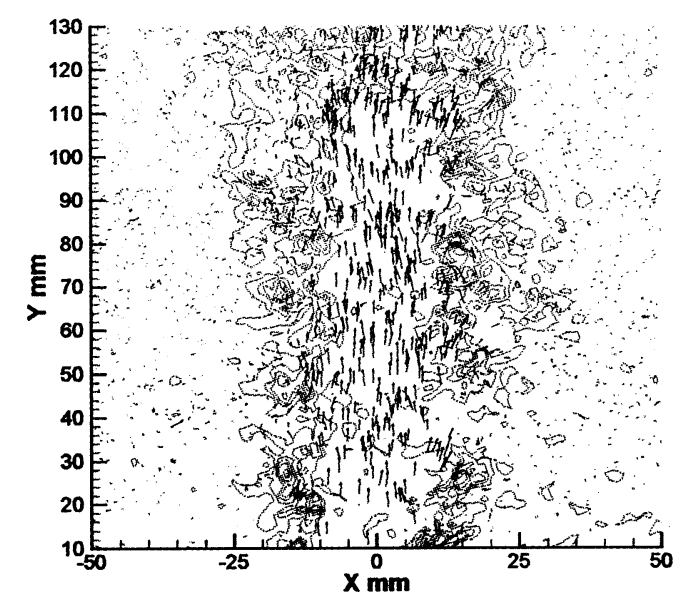

Figure 8. Example of certain instantaneous PIV and PTV result

\section{CONCLUSION}

In the present study, an optical based simultaneous measurement system for liquid-solid two-phase flow is developed. By utilizing the Rhodamine B fluorescent tracers and optical filters, the images of the tracer particles and test particles are separated successfully by the recording system shown as figure 2 . The improved PIV and PTV techniques are applied to the processing of the images of the tracers and test particles, respectively, to improve the spatial resolution and the accuracy of the velocity analyzing of both phases. The system was used to measure velocities of both phases in the near field of a liquid-solid circular jet flow simultaneously, and the experimental results are as follows.

1. There is no obvious modification of the averaged velocity of the continuous phase when the larger test particles are added in the flow with low loading rate.

2 . The turbulence intensity of the continuous phase was increased with the loading of the test particles larger than the Kolmogorov length scale.

3. The preferential concentration of the particles by turbulence is proved.

The present experimental results agreed with the results of previous numerical simulations and experimental research. We can conclude that the present combined PIV-PTV measurement system is effective to measure the velocities of both phases simultaneously for the liquid-solid two-phase flow.

\section{REFERENCES}

1. Suzuki, Y. and Mikami, F., Application of 3D-PTV to the Measurements of Hydrodynamically Interacting Particles. $6^{\text {th }}$ Proceedings, Kanto Branch of JSME(2000-3), PP169170.

2. Hishida, K.and Hanzawa,A., Turbulence Structure of Liquid-Solid Two-Phase Channel Flow( $1^{\text {st }}$ Report. Measurements of Two-Phase Flow by DPIV), JSME B 62 593(1996-1) PP18-25.

3. Suzuki,Y. and Ikenoya, M., There-dimensional PTV Measurement of the Phase Relationship Between Coherent Structures and Dispersed Particles in a Turbulent Channel Flow, Proceedings of the third international workshop on PIV'99 - Santa Barbarra, PP107-112.

4. Kiger, K.T. and Pan, C., Two-phase PIV for Dilute Solid/Liquid Flow, Proceedings of the third international workshop on PIV'99 - Santa Barbarra, PP157-162.

5. Hart, D.P. Super-Resolution PIV by Recursive LocalCorrelation, Journal of Visualization, Vol.10, 1999.

6. Saga, T. and Segawa, S., Evaluation on the performance of correlation based PTV method, $6^{\text {th }}$ Proceedings, Kanto Branch of JSME(2000-3), PP171-172.

7. Squires, K.D. and Eaton J.K., Preferential concentration of particles by turbulence, Phys. Fluids, A 3(5), 1991, PP1169-1178. 